'Departamento de Kinesiología Universidad Católica del Maule, Talca, Chile. 2Laboratorio de Envejecimiento y Funcionalidad. Facultad de Ciencias de la Salud de la Universidad Católica del Maule, Talca, Chile.

${ }^{3}$ Laboratorio de Función Ventilatoria. Facultad de Ciencias de la Salud de la Universidad Católica del Maule, Talca, Chile.

aMagíster en Kinesiología.

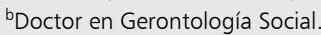
'Magíster en Ciencias, mención Patología.

dMagíster ( en Kinesiología.

Recibido el 26 de junio de 2014, aceptado el 29 de marzo de 2015.

Correspondencia a: Paul Medina González Departamento de Kinesiología, Facultad de Ciencias de la Salud, Universidad Católica del Maule Av. San Miguel № 3605. Talca, Chile.

Teléfono: +56712413622

Fax: +56712203399 pmedina@ucm.cl

\section{Distancia recorrida y costo fisiológico según el nivel socioeconómico y género durante la prueba de caminata en seis minutos en adultos mayores autovalentes de la ciudad de Talca}

\author{
PAUL MEDINA G. ${ }^{1,2, \mathrm{a}}$, ELADIO MANCILLA S..$^{1,2, \mathrm{~b}, \mathrm{c}}$, \\ RODRIGO MUÑOZ C. ${ }^{1,3, \mathrm{~d}}$, MÁXIMO ESCOBAR C. . $^{1,3, \mathrm{a}}$
}

\section{Effects of gender and socioeconomic status of older people on the execution of the 6 minute walk test}

Background: The six minute walk test (6MWT) is an important physical performance measure used in older people. Aim: To assess the total distance walked and the physiological cost of the 6MWT in non-disabled older people. Material and Methods: Fifty six females aged $69 \pm 5$ years with a body mass index (BMI) of $31 \pm 4 \mathrm{~kg} / \mathrm{m}^{2}$ and sixteen males aged $70 \pm 7$ years with a BIM of $29 \pm 4 \mathrm{~kg} / \mathrm{m}^{2}$ underwent a $6 \mathrm{MWT}$. The total distance walked was registered and the physiological cost (PC) of the test was calculated as the ratio between the difference of heart rate at work and at rest and the walking speed. The socioeconomic status (SES) of participants was determined using a questionnaire designed in Chile. Results: Among participants of middle-lower and middle-upper SES, the $P C$ was $0.43 \pm 0.1$ and $0.44 \pm 0.09$ beats $/ \mathrm{min}$, respectively, $p=N S$. Males of middle-upper SES had a significantly higher PC than their middle-lower SES counterparts. The total distance walked was $493 \pm 58$ and $501 \pm 63 \mathrm{~m}$ among participants of upper and lower SES. The plateau in reserve heart rate appeared earlier among participants of upper SES of both genders. Conclusions: Older participants of middle-upper SES had a higher physiological efficiency during the execution of the 6MWT.

(Rev Med Chile 2015; 143: 484-492)

Key words: Aging; Exercise test; Monitoring, physiologic; Socioeconomic factors.

\footnotetext{
L
} a transición demográfica hacia una estructura poblacional envejecida es una situación evidente para poblaciones en vías de desarrollo, al respecto, América Latina ha experimentado un aumento sostenido de la esperanza de vida, llegando a 73,4 años ${ }^{1}$. Esta situación se traduce en un incremento cuantitativo de la población de adultos mayores $(\mathrm{AM})^{2}$, que desde una perspectiva de desarrollo humano tienen requerimientos específicos, los cuales desafían el planteamiento de políticas públicas atingentes hacia el mantenimiento y mejoras en la calidad de $v$ ida $^{3}$, considerando para esto la implementación efectiva de planes que consoliden el envejecimiento activo y saludable ${ }^{4}$.

Se ha documentado que la determinación de la capacidad funcional es la manera más pertinente para caracterizar la salud y calidad de vida 
de los $\mathrm{AM}^{5}$. En este escenario, la expresión de movimiento considerando la postura bípeda y su correspondiente estado dinámico de marcha, resultan fundamentales como hitos de función motora, tanto para su desarrollo en las primeras etapas del ciclo vital ${ }^{6}$, como durante el proceso de envejecimiento avanzado, en el que condiciona y traduce diferentes niveles de independencia y autonomía ${ }^{7}$.

La herramienta más utilizada en la clínica para determinar la capacidad funcional en sujetos es la prueba de caminata en seis minutos (PC6m), la cual reporta investigaciones para diferentes contextos etarios ${ }^{8,9}$ y funcionales ${ }^{10-12}$. Sin embargo, en la población de AM, el enfoque predominante del análisis clínico se centra en el rendimiento obtenido en metros, así como el comportamiento de frecuencia cardiaca (FC), sensación subjetiva de fatiga (SSF) y saturación de oxígeno $\left(\mathrm{SpO}_{2}\right)$ antes y después de la prueba ${ }^{9,13}$, dejando en una sistemática interrogante el costo fisiológico temporal durante cada minuto que involucra la ejecución de esta tarea aeróbica.

El gasto energético de la marcha humana ha sido frecuentemente utilizado como un traductor de eficiencia fisiológica ${ }^{14}$, al respecto, las mediciones de referencia proponen valorar cuantitativamente el costo fisiológico a través de pruebas que consideran como indicador el consumo de oxígeno mediante análisis de gases espirados ${ }^{15,16}$. Sin embargo, en la práctica clínica esta propuesta tiene dificultades de aplicabilidad, debido a los altos costos que significa implementarla y principalmente la falta de confort para el participante al momento de utilizarla. Existen alternativas que cumplen con la propiedad clinimétrica de aplicabilidad, manteniendo niveles aceptables de confiabilidad ${ }^{17}$ y valide $z^{17,18}$. Para tal contexto, se incluyen herramientas que miden el gasto energético de la marcha mediante el comportamiento de la frecuencia cardiaca de trabajo ( $\mathrm{FCw}$ ) en relación a sus niveles basales. $\mathrm{Al}$ respecto, se ha propuesto en la literatura el índice de costo fisiológico (ICF) como un indicador, el cual, considera para el análisis el comportamiento de la FCw y la velocidad de marcha, reportando resultados para investigaciones realizadas en diferentes poblaciones y contextos funcionales ${ }^{17-21}$.

Existe evidencia que el ambiente tendría influencia sobre la expresión de funcionalidad y salud en $\mathrm{AM}^{22}$. Algunos artículos relacionan de manera inversa y significativa aspectos ecológicos pertinentes al hombre contemporáneo tales como nivel de ingresos y educación, con la manifestación de limitaciones funcionales ${ }^{23-25}$. En este escenario, Chile es un ejemplo de inequidades sociales y económicas lo que se traduce en menores niveles de salud en los quintiles más bajos de la población ${ }^{26}$, además esta brecha aumenta con el proceso de envejecimiento, reportándose mayores tasas de discapacidad para niveles socioeconómicos (NSE) bajos $^{27}$. El propósito de la presente investigación es evaluar el rendimiento en metros y costo fisiológico de la PC6m según estratificación socioeconómica en una muestra de AM autovalentes pertenecientes a los sectores norte y oriente de la ciudad de Talca-Chile.

\section{Material y Método}

\section{Participantes}

En esta investigación observacional y transversal, participaron mediante un muestreo por conveniencia y no probabilístico, 72 AM provenientes de instituciones sociales de la comunidad pertenecientes a los sectores norte y oriente de la ciudad de Talca-Chile; los participantes firmaron un consentimiento que cumplió con las normas propuestas en la Declaración de Helsinki y fue aprobado por el Comité de Ética Científico de la Universidad Católica del Maule (informe de seguimiento $\mathrm{N}^{\circ} 2 / 2014$ ). Los criterios de inclusión fueron controlados mediante la aplicación del Examen de Medicina Preventiva del Adulto Mayor $(\text { EMPAM })^{28}$, verificando edad entre 60-75 años, antropometría específica de normopeso o sobrepeso (Estadímetro DETECTO, modelo 2392), autovalentes según la Evaluación Funcional del Adulto Mayor-Chile, parte A (EFAM-Chile), nivel cognitivo normal (Test Minimental abreviado $\geq 13$ puntos) y sin depresión establecida (Escala de Yessavage $<5$ puntos). Por su parte, fueron excluidos los sujetos con enfermedades crónicas descompensadas, riesgo de caídas, secuelas de enfermedades neurológicas o cardiovasculares y dolor moderado de miembros inferiores. El NSE se determinó mediante la aplicación de la encuesta ADIMARK $^{29}$, en este sentido se hizo operacional el NSE en dos grupos: a) medio-bajo (MB) para los estratos C3 y D ( $\mathrm{n}=40,30$ mujeres $)$ y b) NSE medio-alto (MA) para Abc1 y C2 $(\mathrm{n}=32,26$ mujeres). 
Las características generales de los participantes se presentan en la Tabla 1.

\section{Procedimiento}

Posterior a la caracterización inicial, se determinó el comportamiento fisiológico basal a través de FC (Polar, FS3 GRY, USA/CAN), frecuencia respiratoria, presión arterial (esfigmomanómetro digital, Omron, modelo Hem-7114), SSF ${ }^{30}$ (escala de Borg modificada) y percepción de dolor (escala visual análoga), Tabla 2.

Dos evaluadores (evaluador $1=\mathrm{E} 1$; evaluador 2 = E2) otorgaron las instrucciones pertinentes y solicitaron la ejecución de la PC6m en un circuito elíptico de setenta metros, el que se demarcó para la identificación de cada metro, lo que permitió el registro de la distancia cada 15 segundos, por su parte, el control temporal de la FCw se obtuvo mediante un telémetro. Finalmente, se ubicaron conos para establecer los límites de la pista (Figura 1). Cabe destacar que la utilización de un circuito elíptico, tiene el propósito de garantizar el blanco de acción aeróbico evitando mecanismos neuromotores de desbalance e inestabilidad dados por abruptas aceleraciones y desaceleraciones, lo cual en AM es particularmente sensible ${ }^{31}$.

Ambos evaluadores controlaron el tiempo simultáneamente mediante un cronómetro (CASIO, HS-3 Basic Trainer V. 1.2.0), al respecto el E2 se encargó de registrar cada 15 segundos la FCw, por su parte, el E1 se encargó de registrar la distancia recorrida cada 15 segundos e incentivar la ejecución correcta de la prueba para garantizar la sinceridad del esfuerzo y el trabajo de caracte-

Tabla 1. Características demográficas y antropométricas de los participantes (n total = 72)

\begin{tabular}{|lllccccc|}
\hline NSE & Género & $\mathbf{n}$ & $\begin{array}{c}\text { Edad } \\
\text { (años) }\end{array}$ & $\begin{array}{c}\text { Peso } \\
\text { (kilos) }\end{array}$ & $\begin{array}{c}\text { Talla } \\
\text { (metros) }\end{array}$ & $\begin{array}{c}\text { IMC } \\
\text { (Kilos/m } \mathbf{m}^{\mathbf{2})}\end{array}$ & $\begin{array}{c}\text { EFAM A } \\
\text { (puntaje) }\end{array}$ \\
MB & F & 30 & $69 \pm 4$ & $71,8 \pm 9,8$ & $1,52 \pm 0,06$ & $31,2 \pm 4,3$ & $49 \pm 3$ \\
& M & 10 & $68 \pm 6$ & $82,4 \pm 12,7$ & $1,64 \pm 0,05$ & $30,5 \pm 3,1$ & $51 \pm 2$ \\
& Total & 40 & $69 \pm 5$ & $74,1 \pm 11,3$ & $1,54 \pm 0,07$ & $31,0 \pm 4,0$ & $49 \pm 3$ \\
MA & F & 26 & $70 \pm 6$ & $70,7 \pm 13,0$ & $1,53 \pm 0,06$ & $30,2 \pm 4,7$ & $52 \pm 3$ \\
& M & 6 & $74 \pm 7$ & $81,4 \pm 10,6$ & $1,69 \pm 0,07$ & $28,5 \pm 4,2$ & $52 \pm 2$ \\
& Total & 32 & $71 \pm 6$ & $72,9 \pm 13,2$ & $1,56 \pm 0,09$ & $29,8 \pm 4,6$ & $52 \pm 3$ \\
\hline
\end{tabular}

Los valores se expresan en promedios \pm desviación estándar para cada variable. NSE $=$ Nivel Socioeconómico; $\mathrm{MB}=\mathrm{Medio}$ bajo; $M A=$ Medio alto; $F=$ Femenino; $M=$ Masculino y $n=$ número de participantes por grupo. EFAM A = Evaluación Funcional del Adulto Mayor parte A. El modelo de comparación de medias no expresa diferencias significativas para las variables descritas según la intersección de los factores NSE y género.

Tabla 2. Características fisiológicas basales de los participantes ( total = 72)

\begin{tabular}{|llccccccc|}
\hline NSE & Género & $\mathbf{n}$ & $\begin{array}{c}\text { FC } \\
\text { (lat/min) }\end{array}$ & $\begin{array}{c}\text { FR } \\
\text { (v/min) }\end{array}$ & $\begin{array}{c}\text { PAS } \\
(\mathbf{m m} \text { Hg) }\end{array}$ & $\begin{array}{c}\text { PAD } \\
(\mathbf{m m ~ H g )}\end{array}$ & $\begin{array}{c}\text { Dolor } \\
(\mathbf{0}-10)\end{array}$ & $\begin{array}{c}\text { SSF } \\
(\mathbf{0}-\mathbf{1 0})\end{array}$ \\
$\mathrm{MB}$ & $\mathrm{F}$ & 30 & $70 \pm 10$ & $18 \pm 3$ & $133 \pm 15$ & $76 \pm 10$ & $1 \pm 2$ & $1 \pm 1$ \\
& M & 10 & $68 \pm 12$ & $17 \pm 3$ & $156 \pm 20$ & $85 \pm 9$ & $0 \pm 0$ & $0 \pm 0$ \\
& Total & 40 & $69 \pm 10$ & $18 \pm 3$ & $138 \pm 19$ & $78 \pm 11$ & $1 \pm 2$ & $0 \pm 1$ \\
MA & F & 26 & $68 \pm 9$ & $17 \pm 4$ & $137 \pm 22$ & $74 \pm 8$ & $1 \pm 3$ & $0 \pm 0$ \\
& M & 6 & $71 \pm 10$ & $16 \pm 4$ & $153 \pm 27$ & $73 \pm 11$ & $0 \pm 0$ & $0 \pm 0$ \\
& Total & 32 & $68 \pm 10$ & $17 \pm 4$ & $141 \pm 24$ & $73 \pm 9$ & $1 \pm 2$ & $0 \pm 0$ \\
\hline
\end{tabular}

Los valores se expresan en promedios \pm desviación estándar para cada variable. NSE = Nivel Socioeconómico; MB = Medio bajo; $M A=$ Medio alto; $F=$ Femenino; $M=$ Masculino; $n=$ número de participantes por grupo; $F C=$ frecuencia cardiaca; $F R=$ frecuencia respiratoria; PAS = presión arterial sistólica; PAD = presión arterial diastólica; SSF = sensación subjetiva de fatiga; lat = latidos; $v$ = ventilaciones y $\min =$ minuto. El modelo de comparación de medias no expresa diferencias significativas para las variables descritas según la intersección de los factores NSE y género. 


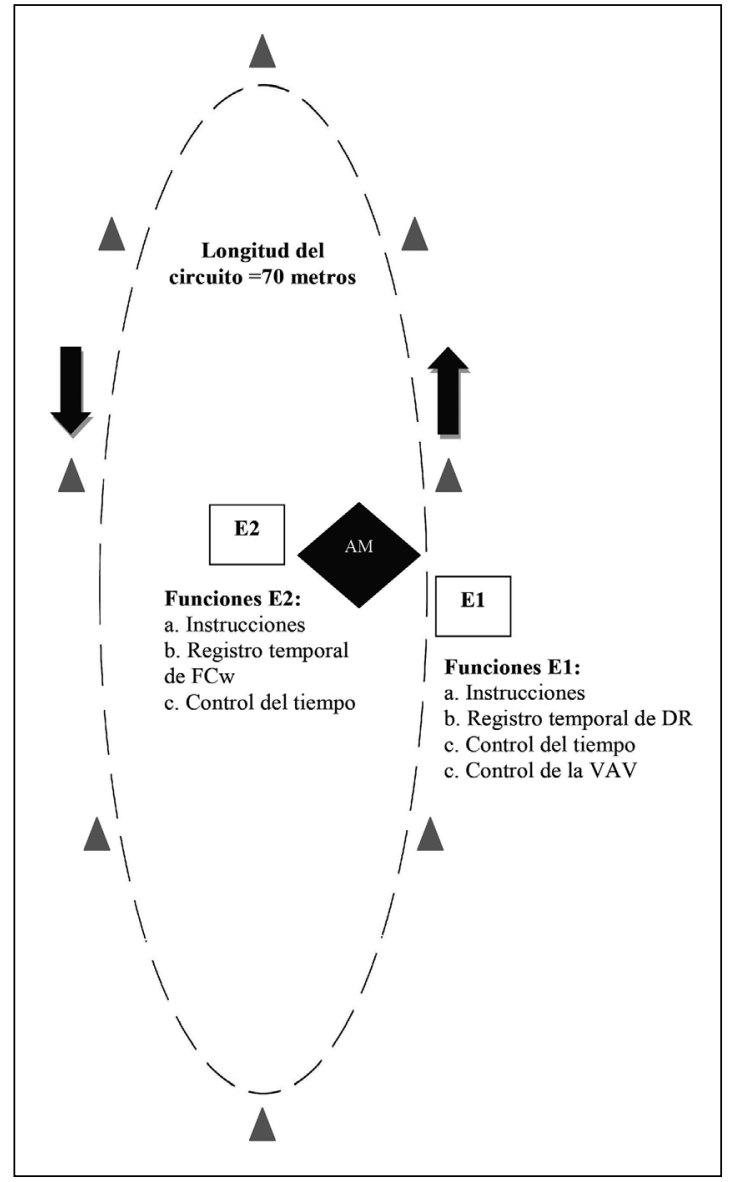

Figura 1. Planificación del circuito elíptico correspondiente a la ejecución de la PC6m. La línea discontinua representa el circuito. $\mathrm{E} 1$ = evaluador $1, \mathrm{E} 2$ = evaluador $2, \mathrm{AM}=$ adulto mayor participante, FC $w$ =frecuencia cardiaca de trabajo, $\mathrm{DR}=$ distancia recorrida y $\mathrm{VAV}=$ ventana de acción valorativa. Las flechas negras indican la dirección del movimiento y los triángulos grises representan los conos de demarcación del circuito. Nota: la demarcación no está desarrollada a escala.

rísticas predominantemente aeróbico. La estandarización del incentivo durante la ejecución de la PC6m consideró una ventana de acción valorativa (VAV) consecuente con los desempeños fisiológicos y perceptuales reportados en este grupo etario $^{13,16}$. En consecuencia, la VAV contempló particularmente una $S S F \geq 2 / 10 y \leq 7 / 10$, en tanto que el porcentaje de frecuencia cardiaca de reserva utilizada ( $\%$ FCRu) fue $\geq 40 \%$ y $\leq 70 \%$. Complementariamente se indicó al participante ejecutar una adecuada coordinación de la respiración ${ }^{32}$ y tolerar la fatiga periférica generada por la muscu- latura dorsiflexora de tobillo ${ }^{33}$. Datos fuera de la VAV condicionaron el fin y la repetición posterior de la prueba.

El ICF se determinó cada 15 segundos mediante la siguiente fórmula de cálculo: $\mathbf{I C F}=(\mathbf{F C w}-$ $\mathbf{F C b}) / \mathbf{v}$. En donde, ICF =índice de costo fisiológico (latidos/metros), $\mathrm{FCw}=$ frecuencia cardiaca de trabajo (latidos/minuto), $\mathrm{FCb}=$ frecuencia cardiaca basal (latidos/minuto) y v =velocidad de marcha (metros/minuto). Para el comportamiento temporal del \%FCRu, se definió como punto crítico fisiológico (PCF) el momento en el cual se alcanzan diferencias significativas desde el reposo.

\section{Análisis estadístico}

El manejo descriptivo de las variables fue mediante promedio y \pm 1 desviación estándar. Para determinar si existen diferencias en el comportamiento de variables demográficas, antropométricas y fisiológicas de base según la intersección de los factores NSE y género, se realizó un modelo de comparación de medias.

Para la comparación del rendimiento en metros y el costo fisiológico de marcha según NSE y género se utilizó la prueba $t$ de Student para muestras independientes. El nivel de significación estadística se estableció en un $\mathrm{p} \leq 0,05$. Los programas que se utilizaron para el manejo de los datos fueron el SPSS versión 18.0 y GraphPad Prism versión 5.0.

\section{Resultados}

El comportamiento del ICF de marcha según el NSE y género se reporta en la Figura 2. En este contexto, la Figura $2 \mathrm{~A}$ muestra que el promedio de este indicador es significativamente superior en hombres del NSE MB en relación al alto $(\mathrm{p}=0,046)$. Complementario a esto, la representación del costo fisiológico cada 15 segundos expone que el estado estacionario fisiológico lo alcanza el grupo de AM pertenecientes al NSE MA (Figura 2C) antes que su contraparte (Figura 2B).

Considerando el rendimiento en metros y su correspondiente utilización porcentual de frecuencia cardiaca de reserva, se reportan los resultados obtenidos durante la ejecución de la PC6m (Figura 3). El rendimiento en metros obtenido durante la PC6m fue cercano a 500 metros para ambos NSE operacionalizados, no existiendo 


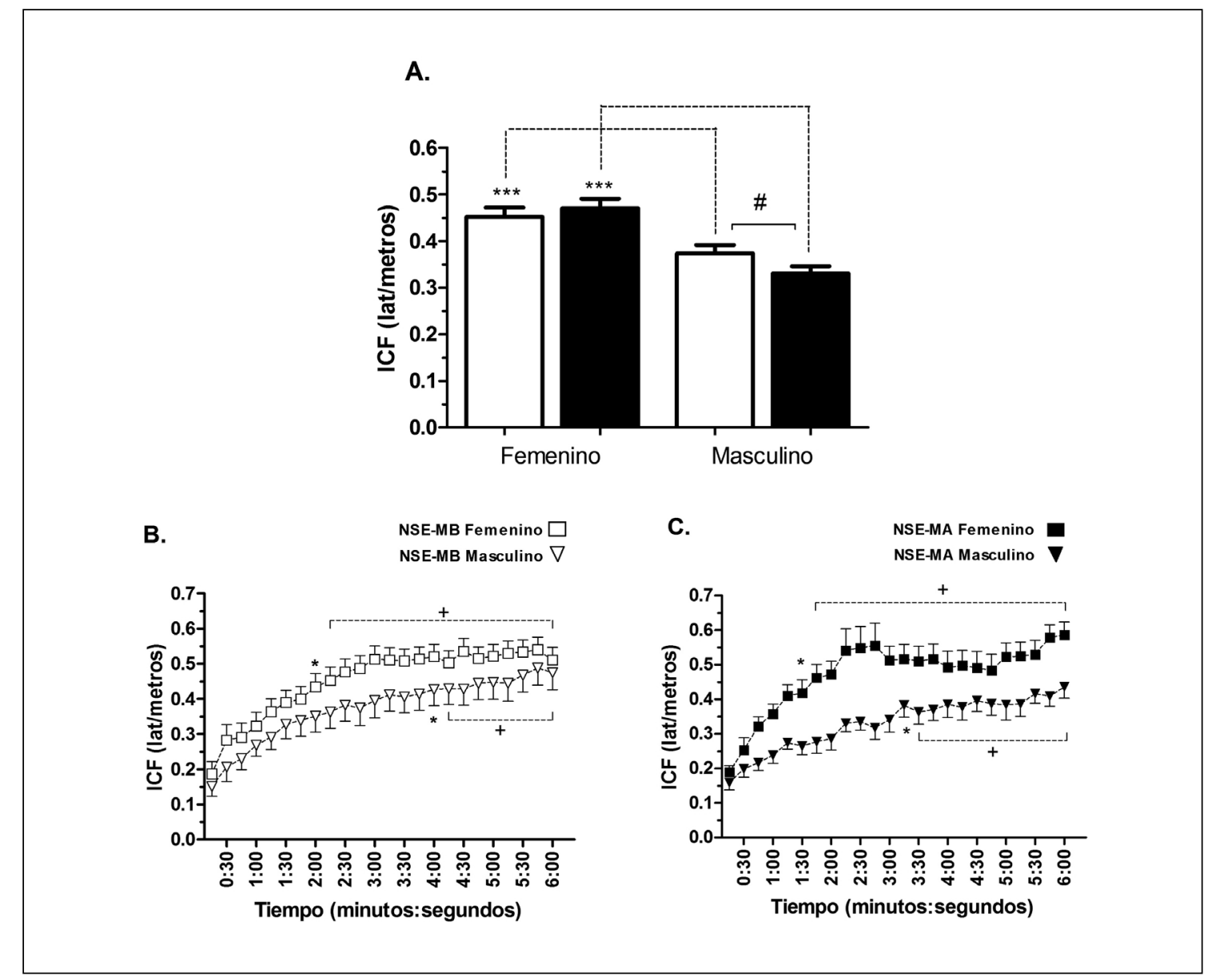

Figura 2. Comportamiento del ICF durante la PC6m según NSE y género. A) Promedio y \pm SEM del costo fisiológico de la PC6m según NSE y género para la totalidad de participantes NSE $M B, n=40(F=30$ y $M=10)$ y NSE $M A, n=32(F=26$ y $M=6)$. ${ }^{* *} p<0,001$ al comparar el ICF según género $y{ }^{*} p<0,05$ al comparar ICF según NSE para el género masculino; B) Comportamiento del promedio y \pm SEM del ICF durante 6 min de marcha máxima, las cajas representan el registro cada 15 segundos según el género en el NSE MB y C) NSE MA. ${ }^{*} p<0,05$ respecto del minuto 0:15 para ambos géneros y NSE. ${ }^{+}$Estado estacionario fisiológico para ambos géneros y NSE. El color blanco representa el NSE MB, mientras que el color negro representa el NSE MA. Los cuadrados representan las mujeres y los triángulos invertidos los hombres.

diferencias significativas (Figura 3A), por su parte, el análisis según género si reporta diferencias estadísticamente significativas ( $\mathrm{p}<0,001)$, siendo menor en las mujeres de ambos NSE (Figura 3A). En este contexto, los hombres del NSE MB tuvieron un rendimiento superior en 21 metros, mientras que en las mujeres este fue superior en el NSE MA con una distancia recorrida mayor en 21 metros (Figura 3A). Al observar los comportamientos de las curvas de trabajo (Figuras 3B, 3C y 3D), estas presentan mediante el \%FCRu el punto de inicio para el estado estacionario y el punto de recuperación crítico (ver leyenda Figura 3). Al respecto, alrededor del primer minuto posterior al inicio de marcha, se logra el estado estacionario fisiológico siendo temporalmente anterior en el NSE MB (Figura 3B), este comportamiento cambia cuando se analiza según género, en donde las mujeres del NSE MA alcanzan antes esta situación fisiológica (Figura 3C). Además, se aprecia una forma de la curva de trabajo diferente según género, la cual es independiente del NSE (Figura 3C y Figura 3D). 
A.

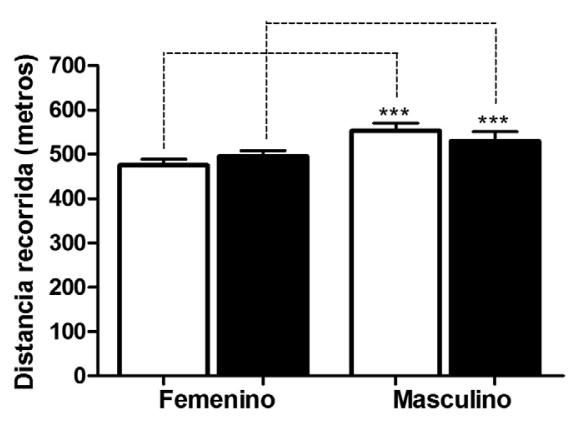

C.

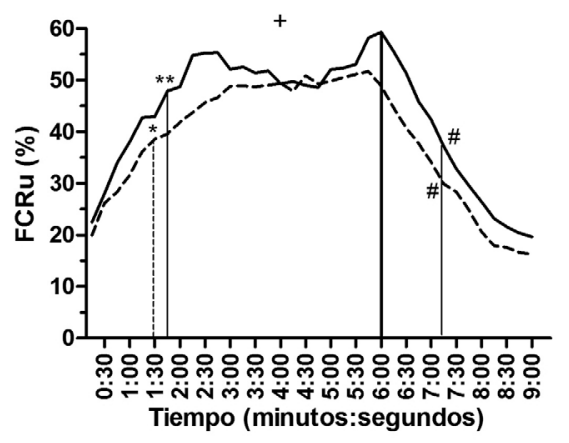

B.

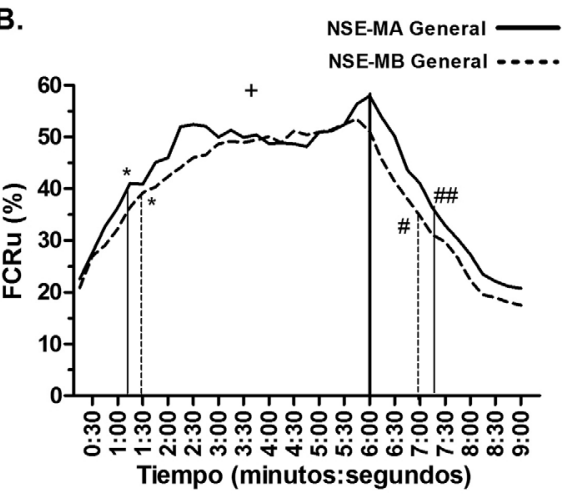

D.

NSE-MA Masculino

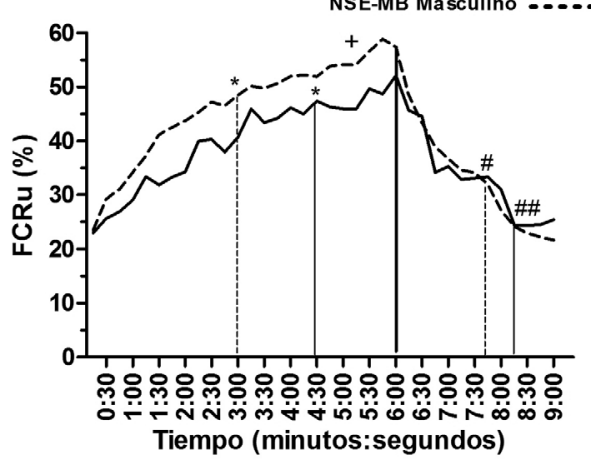

Figura 3. Comportamiento de la DR y \%FCRu según NSE y género durante la PC6m. A) Promedio y \pm SEM de la DR en la PC6m según NSE y género, ${ }^{* *} p<0,001$; B) Promedio del registro cada 15 segundos del \%FCRu de trabajo y reposo según NSE general; $\mathbf{C})$ según NSE en género femenino y $\mathbf{D})$ según NSE en género masculino. ${ }^{*} p<0,05 y * * p<0,01$ respecto del minuto 0:15 para ambos NSE. ${ }^{*} p<0,05 y{ }^{\# \#} p<0,01$ recuperación respecto del minuto seis de trabajo. ${ }^{+}$estado estacionario fisiológico para ambos NSE. La línea sólida representa el NSE MB, por su parte, la línea interrumpida el NSE MA.

\section{Discusión}

El análisis del costo fisiológico ante demandas de trabajo que se acercan a una máxima capacidad ha sido un foco frecuente de estudio ${ }^{15}$. Se dispone de información para diferentes pruebas que valoran este comportamiento en contextos como: bicicleta estática, cinta rodante y marcha en pasillo ${ }^{35}$. Estas miden principalmente consumo de oxígeno, sin embargo, se ha reportado la utilidad que ofrece la frecuencia cardiaca como indicador complementario de costo fisiológico para marcha que se acerca a máximos requerimientos ${ }^{18,36}$. Cabe mencionar, que este tipo de análisis han generado diversos protocolos para exigir pertinentemente al sistema cardiovascular en un contexto clínico.

Desde el punto de vista del ICF para marcha máxima (velocidad máxima del sujeto durante la ejecución de la PC6m), existe una experiencia documentada por Danielsson et al, en la que evaluaron el costo fisiológico durante la ejecución de la PC6m según norma ATS en sujetos con secuela de accidente cerebrovascular, reportando resultados cercanos a $0,6 \pm 0,41$ latidos $/ \mathrm{m}^{37}$. Estos resultados son superiores a los del presente estudio (Figura 2A) debido a que se trata de sujetos con compromiso neuromotor, lo que se traduce en una distancia total de $352 \pm 136$ metros, contrastando desde el punto de vista de eficiencia fisiológica y mecánica con los resultados obtenidos en la pre- 
sente investigación. En tal contexto, el ICF sería un indicador sensible a procesos que involucran ineficacia mecánica y fisiológica de la marcha; tal aseveración concerniente a la validez de tipo discriminativa, se fortalece al comparar los resultados del ICF obtenidos en nuestra investigación, los cuales sobrepasan en aproximadamente $75 \%$ lo reportado para marcha confortable en sujetos asintomáticos ${ }^{19}$. Además, siendo esta la primera experiencia que reporta el comportamiento del ICF durante marcha de máxima exigencia en AM autovalentes, pensamos que los datos obtenidos podrían orientar como referencia el análisis del costo fisiológico en situaciones clínicas y de investigación. El comportamiento general del ICF durante la ejecución de la PC6m no expresó diferencias según el NSE, esto se podría explicar por la condición de autovalencia similar de los sujetos que participaron en este estudio (Tabla 1), lo que conjuntamente a la velocidad promedio alcanzada les permitiría desempeñarse de manera hábil y eficiente en dicho contexto funcional ${ }^{38}$. No obstante, al analizar los datos según el género, el NSE como factor presentó diferencias a favor del MB para el género masculino (Figura 2A). Esta situación se podría explicar por las experiencias laborales de los $\mathrm{AM}^{23-25}$, donde la mayoría de los sujetos del NSE MB tendrían trabajos de requerimientos físicos y que demandarían traslados frecuentes en terrenos irregulares, mientras que los del NSE MA preferentemente se desempeñarían en trabajos de oficina, con un requerimiento más cognitivo asociado a la toma de decisiones. En este escenario, pareciera que el contexto laboral impacta en el costo fisiológico de la marcha máxima de AM de género masculino, al respecto resulta pertinente considerar que la distancia recorrida también fue mayor en este grupo (Figura 3A), lo que se podría traducir en un mayor consumo de oxígeno ${ }^{15,16} \mathrm{y}$ por tanto una amplia adaptabilidad funcional para aquellos que lo demandaron con mayor énfasis en etapas anteriores de su ciclo vital. Es probable, que la diferencia etaria entre los NSE operacionalizados para el género masculino (6 años mayor en el NSE MA, Tabla 1), además del bajo número de hombres participantes podrían haber sesgado los resultados obtenidos, por lo que deben ser tomados con cautela además de comprobarse en próximas experiencias.

Los resultados del rendimiento en metros obtenido durante la PC6m fueron consistentes con los reportados como referencia para grupos con características demográficas similares ${ }^{13}$. Al respecto, el mayor costo fisiológico asociado a un menor rendimiento en metros para el género femenino es concordante con experiencias anteriore ${ }^{15}$ y se podría explicar por el proceso de reserva funcional y fisiológica el cual sería divergente según el género ${ }^{34}$.

Desde el punto de vista de la monitorización del costo fisiológico, durante la ejecución de la PC6m, las diferencias reportadas en la forma de la curva de trabajo traducida por el \%FCRu invitan a plantearnos interrogantes acerca de la autorregulación de la carga de trabajo. Esta podría depender de factores asociados al género tales como motivación o sinceridad del esfuerzo. La aparición del PCF tiene diferentes temporalidades según el NSE (Figuras 3B, 3C y 3D), este comportamiento indica que el NSE tendría un impacto en la capacidad adaptativa fisiológica ante demandas de esfuerzo elevadas, la explicación a este fenómeno podría ser por dos vías: a) calidad de la indumentaria condicionada por el poder adquisitivo de los individuos ${ }^{24} \mathrm{y}$ b) hábitos de alimentación los cuales son significativamente favorables hacia los NSE más altos ${ }^{39}$. Complementando lo anterior y en el contexto de aparición de compromisos metabólicos asociados a elementos multifactoriales, entre ellos el nutricional, se ha reportado a los niveles séricos de bicarbonato como un posible factor que en condiciones descendidas $(<23 \mathrm{mEq} / \mathrm{L})$, explicarían una menor velocidad de marcha y fuerza muscular debido a la aparición de acidosis metabólica y su consiguiente catabolismo proteico $^{40}$. No obstante, en el presente trabajo no se evidencian diferencias significativas en la velocidad de marcha lo que invita a profundizar esta temática en futuras investigaciones considerando indicadores a nivel molecular y celular junto a la expresión de variables más sensibles a esta realidad tales como el ritmo de desarrollo de fuerza de extensores de rodilla y el análisis cinético de marcha.

Dentro de las limitaciones de la investigación exponemos que no se utilizaron por separado las categorías socioeconómicas descritas en el instrumento original ${ }^{29}$, además la muestra no es representativa de la población y el número de sujetos hace incipiente el análisis según género. Por último, una de las explicaciones más contundentes a los resultados obtenidos para el género masculino emerge de la diferencia de edad entre 
los NSE operacionalizados (Tabla 1), la cual al ser de 6 años se estima como clínicamente significativa para la expresión de fuerza como velocidad en AM japoneses autovalentes ${ }^{41}$. No obstante lo anterior, esta propuesta metodológica forma parte de una línea de trabajo que busca orientar investigaciones focalizadas en el movimiento humano en sus distintos niveles de expresión ${ }^{42}$.

En conclusion, nuestros resultados reflejan que en AM autovalentes de la comunidad, el NSE tendría una influencia dependiente del género para el comportamiento de la DR y costo fisiológico durante la ejecución de una PC6m. Sin embargo, la adaptación temporal fisiológica tiende a una mayor eficiencia en el NSE MA para ambos géneros, por esta razón se recomienda analizar y evaluar esta situación en futuras investigaciones y situaciones profesionales considerando que una mayor profundidad en la información garantizaría mejores decisiones diagnósticas e investigativas.

\section{Referencias}

1. Chackiel J. La dinámica demográfica de América Latina. Serie población y desarrollo - Comisión Económica para América Latina y el Caribe [Internet]. 2004 [Acceso en 2014, enero 20]; 52: 104pp. Disponible en: URL:http://www.eclac.cl/cgibin/getProd.asp?xml=/ publicaciones/xml/0/14860/P14860.xml\&xsl=/celade/ tpl/p9f.xsl\&base=/celade/tpl/top-bottom.xsl.

2. Centro Latinoamericano y Caribeño de DemografíaDivisión de Población de la CEPAL. Manual sobre indicadores de la calidad de vida en la vejez. Capítulo 1: Demografía del envejecimiento. Santiago, Chile: Editado por Naciones Unidas [Internet]. 2006 [Acceso 7 de marzo de 2014]; 164pp. Disponible en: URL:http:// www.eclac.cl/cgibin/getProd.asp?xml=/publicaciones/ $\mathrm{xml} / 0 / 28240 / \mathrm{P} 28240 . \mathrm{xml} \& \mathrm{xsl}=/$ celade/tpl/p9f.xsl\&ba$\mathrm{se}=/$ celade/tpl/top-bottom.xsl

3. Huenchuan S. La protección de salud en el marco de la dinámica demográfica de los derechos. Serie población y desarrollo - Comisión Económica para América Latina y el Caribe [Internet]. 2011 [Acceso 23 de enero de 2014]; 100: 73pp. Disponible en: URL:http://www.eclac.org/ cgi-bin/getProd.asp?xml=/publicaciones/xml/3/43173/ P43173.xml\&xsl=/publicaciones/ficha-i.xsl\&base $=$ / publicaciones/top_publicaciones-i.xsl

4. Arai H, Ouchi Y, Yokode M, Ito H, Uematsu H, Eto F, et al. Toward the realization of a better aged society: messages from gerontology and geriatrics. Geriatr Gerontol Int 2012; 12 (1): 16-22.
5. Lara RA, Mardones MA. Perfil sociodemográfico de la salud y funcionalidad en adultos mayores de la comuna de Chillán. Theoria 2009; 18 (2): 81-9.

6. Hausdorf JM, Zemany L, Peng CK, Golderger L. Maturation of gait Dynamics: stride to stride variability and this temporal organization in children. J Apply Physiol 1999; 86 (3): 1040-7.

7. Jacelon CS. The Barthel Index and Other Indices of Functional Ability. Rehabilitation Nursing 1986; 11: 9-11.

8. Escobar M, López A, Véliz C, Crisóstomo S, Pinochet R. Test de Marcha en 6 Minutos en Niños Chilenos Sanos. Kinesiología 2001; 62: 16-20.

9. Troosters T, Gosselink R, Decramer M. Six-minute walking distance in healthy elderly subjects. Eur Respir J 1999; 14: 270-4.

10. American Thoracic Society Statement. Guidelines for six-minute walk test. Am J Respir Crit Care Med 2002; 166 (march): 111-7.

11. Lisboa C, Barría P, Yáñez J, Aguirre M, Díaz O. La prueba de caminata en seis minutos en la evaluación de la capacidad de ejercicio en pacientes con enfermedad pulmonar obstructiva crónica. Rev Med Chile 2008; 136 (8): 1056-64.

12. López A, Sotomayor L, Álvarez M, Céspedes P, Poblete C, Vásquez P, et al. Rendimiento Aeróbico en Niños Obesos de 6 a 10 Años. Rev Chil Pediatr 2009; 80 (5): 444-50.

13. Osses R, Yáñez J, Barría P, Palacios S, Dreyse J, Díaz $\mathrm{O}$, et al. Prueba de caminata en seis minutos en sujetos chilenos sanos de 20 a 80 años. Rev Med Chile 2010; 138 (9): 1124-30.

14. Saibene F, Minetti A. Biomechanical and physiological aspects of legged locomotion in humans. Eur J Appl Physiol 2003; 88: 297-316.

15. Hossack RF, Bruce RA. Maximal cardiac function in sedentary normal men and women: comparison of age-related changes. J Appl Physiol Respir Environ Exerc Physiol 1982; 53 (4): 799-804.

16. Malatesta D, Simar D, Dauvilliers Y, Candau R, Borrani F, Prefaut $C$, et al. Energy cost of walking and gait instability in healthy 65- and 80-yr-olds. J Appl Physiol 2003; 95 (6): 2248-56.

17. Graham R, Smith N, White C. The reliability and validity of the physiological cost index in healthy subjects while walking on 2 different tracks. Arch Phys Med Rehabil 2005; 86: 2041-6.

18. Danielsson A, Willén C, Sunnerhagen KS. Measurement of energy cost by the physiological cost index in walking after stroke. Arch Phys Med Rehabil 2007; 88 (10): 1298-303. 
19. Cheng-Hsun Wu. Physiological Cost Index of Walking for Normal Adults. Department of Rehabilitation, TenChen General Hospital, Jhongli. Disponible a la fecha 28 de septiembre de 2013 en: www.sped.nutn.edu.tw

20. Fredrickson E, Ruff RL, Daly JJ. Physiological Cost Index as a proxy measure for the oxygen cost of gait in stroke patients. Neurorehabil Neural Repair 2007; 21 (5): 429-34.

21. Ijzerman MJ, Nene AV. Feasibility of the physiological cost index as an outcome measure for the assessment of energy expenditure during walking. Arch Phys Med Rehabil 2002; 83 (12): 1777-82.

22. Ministerio de Salud de Chile. Encuesta Nacional de Salud 2009-2010 [Internet]. 2010 [Acceso 7 de marzo de 2014]. Disponible en: URL:http://web.minsal.cl/portal/ url/item/bcb03d7bc28b64dfe040010165012d23.pd

23. Koster A, Penninx BW, Bosma H, Kempen GI, Harris $\mathrm{TB}$, Newman $\mathrm{AB}$, et al. Is there a biomedical explanation for socioeconomic differences in incident mobility limitation? J Gerontol A Biol Sci Med Sci 2005; 60 (8): 1022-7.

24. Nilsson CJ, Avlund K, Lund R. Onset of mobility limitations in old age: the combined effect of socioeconomic position and social relations. Age Ageing 2011; 40 (5): 607-14.

25. Thorpe RJ Jr, Koster A, Kritchevsky SB, Newman AB, Harris T, Ayonayon $\mathrm{HN}$, et al. Race, socioeconomic resources, and late-life mobility and decline: findings from the Health, Aging, and Body Composition study. J Gerontol A Biol Sci Med Sci 2011; 66 (10): 1114-23.

26. Fundación Pobreza. Brechas Socioeconómicas de la población Chilena. [Internet]. 2004 [Acceso 7 de marzo de 2014]. Disponible en: URL:http://www.superacionpobreza.cl/EditorFiles/File/Umbrales/cap1.pdf

27. Olivares-Tirado P. Perfil Epidemiológico del Adulto Mayor en Chile. Gobierno de Chile. Superintendencia de Salud. Departamento de Estudios y Desarrollo 2006; $1-20$.

28. Ministerio de Salud Chile. Programa de Salud del Adulto Mayor. División de Prevención y Control de Enfermedades. Subsecretaría de Salud Pública. Manual de Aplicación del Examen de Medicina Preventiva del Adulto Mayor (EMPAM). [Internet]. 2014 [Acceso 23 de enero de 2014]. Disponible en: URL:http://www.saludohiggins.cl/attachments/314_Instructivo\%20del\%20 Control\%20de\%20Salud\%200107.pdf

29. Adimark. Mapa socioeconómico de Chile: nivel socioeconómico del país basado en los datos del Censo. Santiago 2000. [Internet]. 2000 [Acceso 26 de julio de
2012]. Disponible en: http://www.adimark.cl/medios/ estudios/mapa_socioeconomico_de_chile.pdf

30. Borg GA. Psychophysical bases of perceived exertion. Med Sci Sports Exerc 1982; 14 (5): 377-81.

31. Wolfson L. Gait and balance dysfunction: a model of the interaction of age and disease. Neuroscientist 2001; 7 (2): 178-83. Review.

32. Rabler B, Kohl J. Coornination-related changes in the rhythms of breathing and walking in humans. Eur J Appl Physiol 2000; 82: 280-8.

33. Segers V, Lenoir M, Aerts P, De Clercq D. Influence of M. tibials anterior fatigue on the walk-to-run and runto-walk transition in non-steady state locomotion. Gait Posture 2007; 25(4): 639-47.

34. Schrack J, Simonsick E, Ferrucci L. The energetic pathway to mobility loss: an emerging new framework for longitudinal studies of aging. J Am Geriatr Soc 2010; 58 (suppl 2): S329-S336.

35. Fletcher GF, Balady GJ, Amsterdam EA, Chaitman B, Eckel R, Fleg J, et al. Exercise standards for testing and training: a statement for healthcare professionals from the American Heart Association. Circulation 2001; 104 (14): 1694-740.

36. Panton LB, Graves JE, Pollock ML, Garzarella L, Carroll JF, Leggett SH, et al. Relative heart rate, heart rate reserve, and $\mathrm{VO} 2$ during sub-maximal exercise in the elderly. J Gerontol A Biol Sci Med Sci 1996; 51 (4): M165-71.

37. Danielsson A, Willén C, Sunnerhagen KS. Physical activity, ambulation, and motor impairment late after stroke. Stroke Res Treat. [Internet]. 2012. [Acceso 23 de enero de 2014]. Disponible en: http://dx.doi. org/10.1155/2012/818513

38. Fritz S, Lusardi M. White paper: "walking speed: the sixth vital sign”. J Geriatr Phys Ther 2009; 32 (2): 46-9.

39. Lallukka T, Laaksonen M, Rahkonen O, Roos E, Lahelma E. Multiple socio-economic circumstances and healthy food habits. Eur J Clin Nutr 2007; 61 (6): 701-10.

40. Abramowitz MK, Hostetter TH, Melamed ML. Association of serum bicarbonate levels with gait speed and quadriceps strength in older adults. Am J Kidney Dis 2011; 58 (1): 29-38.

41. Ishizaki T, Furuna T, Yoshida $Y$, Iwasa $H$, Shimada $H$, Yoshida H, et al.; TMIG-LISA Research Group. Declines in physical performance by sex and age among nondisabled community-dwelling older Japanese during a 6-year period. J Epidemiol 2011; 21 (3): 176-83.

42. Hislop H. The not-so-impossible dream. Tenth Mary McMillan Lecture. Phys Ther 1975; 55 (1): 1069-79. 